\title{
The Elderly Centre Location Problem
}

\author{
Soheil Davari ${ }^{1}$ and Tom Van Woensel ${ }^{2}$ \\ ${ }^{1}$ Hertfordshire Business School, University of Hertfordshire, Hatfield, The United Kingdom \\ ${ }^{2}$ School of Industrial Engineering \& Innovation Sciences, TU Eindhoven, Eindhoven, The Netherlands
}

January 15, 2020

\begin{abstract}
Increased human life expectancy combined with declining birth rates around the globe has led to ageing populations, particularly in the developed world. This phenomenon brings about increased dependency ratios and calls for setting new policies for the elderly citizens. This comprises the provision of a set of life-enhancing services in an accessible and equitable way. In this paper, we consider a multi-period problem of locating senior centres offering these services to the elderly population against budget constraints and capacity limitations. We assume that the attractiveness of facilities to elderlies is inversely proportional with the travel time to access these facilities. Both consistent and inconsistent versions of the problem are considered, aiming at identifying the set of facilities to operate in each region at each period, the service type(s) to be offered and the allocation of budget in each period to location and operation of facilities. A mixed integer mathematical programming model is presented, an efficient iterated local search procedure is proposed and managerial insights are provided.
\end{abstract}

Keywords. Location, Ageing, Multi-period, Public-sector operations research.

\section{Introduction}

In the United Kingdom (UK), the 20th century saw a dramatic transformation of the population pyramid as the changes in fertility, early life and then later life mortality passed into and through the age profile of the population (Leeson et al., 2016). This phenomenon is not limited to the UK. For instance, according to the United Nation's medium population projections, the number of people aged over 65 could rise from just over 600 million in 2015 to close to 2.5 billion by 2100 (Franklin, 2015). In the UK in particular, the Old-Age Dependency Ratio (OADR), which is defined as the number of people over 65 years old for every 1,000 people aged between 16 and 64 years old (ONS, 2017), is estimated to exceed $42 \%$ in 2036 compared to $25 \%$ in 1996 . This change in the demographics of the world has significant implications on a society, such as a transition from infectious and parasitic diseases to non-communicable diseases and chronic conditions (WHO, 2011) as well as a considerable increase in the demand for housing (Government Office for Science, 2016).

The elderly population is the focus of a large number of academic literature ranging from identification of barriers in access to home care services (Suurmond et al., 2016) to using machine learning for recognition of falls (Rescio et al., 2018). One mostly overlooked problem in the literature is locating facilities to provide services for elderlies to cope with the feeling of loneliness which can increase the risk of premature death (Holt-Lunstad et al., 2015) and double the chance of developing Alzheimer's (Wilson et al., 2007). Senior centres are facilities for elderly adults who live independently, providing services such as lunchtime congregate meals, socialisation/entertainment, and referrals to other service agencies (Johnson et al., 2005). There is some evidence in the literature on the correlation of proximity to these facilities and their use, such as elderly visits to exercise facilities (Sallis et al., 1990) and referral to health care services (Varkevisser et al., 2012). Modelling facilities with a distance-sensitive demand is not new to the literature and dates back to the early 60s when Huff (1963) presented a model in which the behaviour of clients in selecting facilities to refer was modelled as an increasing function of the facilities' attractiveness and a decreasing function of the distance to travel. Real-world case studies confirm this assumption of distancesensitivity. For instance, Currie and Reagan (2003) found that each additional mile to the closest hospital corresponds to a $3 \%$ decrease in the probability that a child has a medical check-up. Another study on the contribution of proximity to the attractiveness of facilities was carried out by Zimmerman (1997), which showed that the clients' decisions to have a prostate cancer screening are influenced by the proximity to facilities. Similar studies were carried out by Facione (1999) and Baron et al. (2008).

In many real-world facility location problems, parameters change over time, such as the annual change in demand or even the concentration of people in different districts of a region throughout the day. Owing to this change in demand, existing facilities may be no longer optimal, which necessitates devising a plan to relocate facilities or locate a set of new facilities to be added to the established set of facilities. In some cases, the change in demand can lead to significant costs to the 
network. Another instance of changes in problem parameters is the change of travel times between network nodes. While transportation costs and travel distances may be known in the short-term, in the long-term, changes in network topology, transport technologies and levels of congestion introduce uncertainty regarding future costs, travel times and distances (Daskin et al., 1997).

Community Operations Research (Community OR) is a multi-disciplinary field which gained a lot of interest in the literature. Interested readers can refer to Johnson et al. (2018) for a comprehensive review of these models and how operations research models can be used in a wide range of areas to address challenges such as diversity and inclusion, environmental sustainability and community development. However, there is a paucity of research on using mathematical modelling for senior service design and there are only few studies addressing this. Lin et al. (2012) presented an optimal control model to find the infrastructure capacity of community-based case from a cost-saving viewpoint. Johnson et al. (2005) and Tao et al. (2014) are other publications in the literature dealing with location of facilities for elderlies. They both addressed single period problems neglecting the need to consider spending budget over multiple periods and the need to consider population dynamics. Our paper contributes to the academic literature by considering the multi-period, capacitated and budget-constrained problem of locating facilities for the elderly with consideration of population dynamics. Moreover, we propose an efficient heuristic to solve the problem and provide managerial insights for policymakers. We deal with the problem of multi-period incremental (similar to the terminology used in Albareda-Sambola et al. (2009)) design of hierarchical elderly centre networks with budget and capacity constraints while considering the changes in demographics.

The remainder of the paper is organised as follows. We first give an overview of the recent literature on hierarchical and multi-period facility location problems in Section two. We then present the mathematical model of the problem in Section three. The proposed solution procedure is given in Section four and the computational experiments are reported in Section five. Then, managerial insights are presented in Section six, and conclusions are given in Section seven as well as some avenues for future research.

\section{Literature Review}

Facility location has been an area of interest among academics in the last decade with applications ranging from locating plants (Galli et al., 2018) to retail stores (Marianov et al., 2018). In this section and for the sake of brevity, we do not cover the literature of facility location in detail and instead provide an overview of the literature relevant to our problem. Interested readers in the facility location problem and its variants can refer to comprehensive reviews such as Snyder (2006), Melo et al. (2009), Prodhon and Prins (2014), Knight et al. (2012) and Drezner (2014).

To the best of our knowledge, the first multi-period facility location problem in the literature dates back to 1973 when Warszawski (1973) proposed the concept of multi-dimensional location problems with multiple commodities and stages. Later, some publications addressed similar problems such as Drezner (1995) considering a dynamic $p$-median problem. The multi-period version of facility location models has been addressed relatively less in the literature compared to its singleperiod counterpart. This can be attributed to the more complicated nature of multi-period models which makes solving them considerably more difficult. Publications regarding multi-period facility location problems range from emergency response (Rottkemper et al., 2012; Duhamel et al., 2016) and healthcare (Başar et al., 2011; Schmid, 2012; Sha and Huang, 2012; Zhen et al., 2014; Davari, 2018; Jang et al., 2019) to supply chain management (Hinojosa et al., 2008; Ghahremani-Nahr et al., 2019; Mogale et al., 2018) and electric vehicle charging stations (Chung and Kwon, 2015; Zhang et al., 2017). In terms of solution algorithms, most of the publications have used heuristic and meta-heuristic algorithms such as variable neighbourhood search (Schmid and Doerner, 2010), genetic algorithm (Zhen et al., 2014) and simulated annealing (Fazel Zarandi et al., 2011). Lagrangian relaxation (Marín et al., 2018) has been another popular method to solve multi-period facility location problems as well as decomposition methods (Duhamel et al., 2016).

The hierarchical facility location problem (HFLP) is another variant of facility location problem with applications in healthcare systems (Smith et al., 2009), production-distribution systems (Widener and Horner, 2011), telecommunication networks (Chan et al., 2008) and waste management network design (Barros et al., 1998). Recently, there have been some studies on HFLP such as Wang and Yang (2014) which considered an HFLP in a reverse logistics context and proposed two types of uncertain programming procedures as solution algorithms. In another study, Ou-Yang and Ansari (2017) proposed a hybrid algorithm to solve the HFLP with flow capacity constraints. They applied their model to a case in Jakarta, Indonesia. Shavarani et al. (2018) investigated a futuristic variant of HFLP in which a distance-constrained mobile facility location problem was addressed to locate and launch recharge stations.

From what was said, there is insufficient research into multi-period hierarchical facility location problems in the literature. This paper expands the body of literature and aims to fill this gap by presenting a multi-period hierarchical facility location problem and proposing an efficient heuristic to solve it for instances with up to 50 demand nodes and 20 periods in reasonably short times. 


\section{Mathematical Model}

Our mathematical model builds on Johnson et al. (2005) by considering an incremental establishment of a hierarchical network, inclusion of budget constraints, enforcing capacity restrictions and incorporation of the facility attractiveness into modelling. To describe the problem, we consider a graph with a set of demand nodes $\mathcal{I}=\{1,2, \ldots, m\}$ and a set of potential facilities $\mathcal{J}=\{1,2, \ldots, n\}$ (the sets $\mathcal{I}$ and $\mathcal{J}$ are not necessarily disjoint). Moreover, we assume a set of service types $\mathcal{S}=\left\{1,2, \ldots, l_{\mathcal{S}}\right\}$ and a planning horizon of discrete time periods $\mathcal{T}=\left\{1,2, \ldots, l_{\mathcal{T}}\right\}$. Each demand node $i \in \mathcal{I}$ has a demand for service $k \in \mathcal{S}$ at time $t \in \mathcal{T}$ which is shown as $d_{i k}^{t}$ and each facility has fixed, expansion and operation costs. In each period $t \in \mathcal{T}$, there is a budget $b^{t}$ which must not be exceeded and there is no carry over of the unspent budget possible. Furthermore, each facility $j \in \mathcal{J}$ has a capacity $\lambda_{j k}$ to offer service type $k \in \mathcal{S}$.

We study the problem with a nested hierarchy which means that a higher-level facility provides all services provided by a lower-level facility and at least one additional service (Şahin and Süral, 2007). Moreover, similar to Johnson et al. (2005), we assume that the facility hierarchy is globally inclusive, i.e. a type $k$ facility can provide $k$ and lower levels of services to customers at all nodes. For instance, a centre can be established providing basic services such as services for those with learning disabilities and then expanded to provide services to those with a brain injury resulting from an accident and severe physical disabilities while the learning disability services are maintained. We model the attractiveness of each facility $j \in \mathcal{J}$ to the residents of node $i \in \mathcal{I}\left(a_{i j}\right)$ as a non-increasing function of the travel time $\tau_{i j}$. This attractiveness function can take various forms such as $a_{i j}=\tau_{i j}^{-\eta}$ or $a_{i j}=e^{-\eta \tau_{i j}}$ (used in our paper) where $\eta$ is a parameter which can be tuned using primary research instruments such as questionnaires. We assume that facilities are opened instantaneously between two consecutive periods and once a facility is opened, it must remain operational until the end of the planning horizon (consistent service). An overview of the sets, parameters and decision variables is given below.

$\underline{\text { Sets }}$

$\mathcal{I} \quad$ Set of demand nodes $\{1, \ldots, m\}$

$\mathcal{J}$ Set of potential facility locations $\{1, \ldots, n\}$

$\mathcal{S} \quad$ Set of service types $\left\{1, \ldots, l_{\mathcal{S}}\right\}$

$\mathcal{T}$ Set of periods $\left\{1, \ldots, l_{\mathcal{T}}\right\}$

\section{$\underline{\text { Parameters }}$}

$\begin{array}{ll}d_{i k}^{t} & \text { Demand of type } k \in \mathcal{S} \text { at demand node } i \in \mathcal{I} \text { at time } t \in \mathcal{T} \\ \tau_{i j} & \text { Travel time between demand node } i \in \mathcal{I} \text { and facility } j \in \mathcal{J} \\ a_{i j} \in[0,1] & \text { Attractiveness of facility } j \in \mathcal{J} \text { to the node } i \in \mathcal{I} \text { as a non-increasing function of the travel time }\left(e^{-\eta \tau_{i j}}\right) \\ e_{j h k}^{t} & \text { Expansion cost of facility } j \in \mathcal{J} \text { from service type } h \in \mathcal{S} \text { to service type } k \in \mathcal{S} \text { at time } t \in \mathcal{T} \\ f_{j}^{t} & \text { Fixed cost of locating a facility at } j \in \mathcal{J} \text { at time } t \in \mathcal{T} \\ g_{j k}^{t} & \text { Variable cost of providing service type } k \in \mathcal{S} \text { at node } j \in \mathcal{J} \text { and time } t \in \mathcal{T} \\ b^{t} & \text { Total available budget at time } t \in \mathcal{T} \\ \lambda_{j k} & \text { The capacity of the facility at node } j \in \mathcal{J} \text { to provide service type } k \in \mathcal{S}\end{array}$

$\underline{\text { Variables }}$

$$
x_{j k}^{t}= \begin{cases}1 & \text { If service type } k \in \mathcal{S} \text { is delivered at } j \in \mathcal{J} \text { in period } t \in \mathcal{T} \\ 0 & \text { Otherwise }\end{cases}
$$

$y_{i j k}^{t}:$ The amount of demand of node $i \in \mathcal{I}$ for service $k \in \mathcal{S}$ which is assigned to $j \in \mathcal{J}$ in period $t \in \mathcal{T}$

$$
z_{j h k}^{t}= \begin{cases}1 & \text { If the service type of facility } j \in \mathcal{J} \text { changes from } h \in \mathcal{S} \text { to } k \in \mathcal{S} \text { in period } t \in \mathcal{T} \\ 0 & \text { Otherwise }\end{cases}
$$

The problem is formulated as a mixed-integer model with capacity and budget constraints. In the remainder of this paper, we denote this problem as the Multi-Period Consistent Facility Location Problem with Budget and Capacity constraints 
(MCFLP-BC).

$$
\begin{aligned}
& \text { (MCFLP-BC): max } \sum_{i \in \mathcal{I}} \sum_{j \in \mathcal{J}} \sum_{k \in \mathcal{S}} \sum_{t \in \mathcal{T}} y_{i j k}^{t} \\
& \sum_{k \in \mathcal{S}} x_{j k}^{t} \leq 1 \\
& y_{i j k}^{t} \leq a_{i j} d_{i k}^{t} \sum_{\substack{h \in \mathcal{S} \\
h \geq k}} x_{j h}^{t} \\
& \sum_{j \in \mathcal{J}} y_{i j k}^{t} \leq d_{i k}^{t} \\
& \sum_{i \in \mathcal{I}} y_{i j k}^{t} \leq \lambda_{j k} \\
& \sum_{j \in \mathcal{J}} f_{j}^{t}\left(\sum_{k \in \mathcal{S}} x_{j k}^{t}-\sum_{k \in \mathcal{S}} x_{j k}^{t-1}\right)+\sum_{j \in \mathcal{J}} \sum_{h \in \mathcal{S}} \sum_{k \in \mathcal{S}} e_{j h k}^{t} z_{j h k}^{t}+\sum_{i \in \mathcal{I}} \sum_{j \in \mathcal{J}} \sum_{k \in \mathcal{S}} g_{j k}^{t} y_{i j k}^{t} \leq b^{t} \\
& z_{j h k}^{t} \geq x_{j h}^{t-1}+x_{j k}^{t}-1 \\
& z_{j h k}^{t} \leq x_{j h}^{t-1} \\
& z_{j h k}^{t} \leq x_{j k}^{t} \\
& x_{j k}^{t-1} \leq \sum_{h \in \mathcal{S}} x_{j h}^{t} \\
& x_{j \geq k}^{t} \in\{0,1\} \\
& y_{i j k}^{t} \geq 0 \\
& z_{j h k}^{t} \in\{0,1\}
\end{aligned}
$$$$
\forall j \in \mathcal{J} ; \forall t \in \mathcal{T}
$$$$
\forall i \in \mathcal{I} ; \forall j \in \mathcal{J} ; \forall k \in \mathcal{S} ; \forall t \in \mathcal{T}
$$$$
\forall i \in \mathcal{I} ; \forall k \in \mathcal{S} ; \forall t \in \mathcal{T}
$$$$
\forall j \in \mathcal{J} ; \forall k \in \mathcal{S} ; \forall t \in \mathcal{T}
$$$$
\forall t \in \mathcal{T}: t>1
$$

$$
\begin{array}{r}
\forall j \in \mathcal{J} ; \forall h, k \in \mathcal{S}: h \neq k ; \forall t \in \mathcal{T}: t>1 \\
\forall j \in \mathcal{J} ; \forall h, k \in \mathcal{S} ; \forall t \in \mathcal{T}: t>1
\end{array}
$$$$
\forall j \in \mathcal{J} ; \forall h, k \in \mathcal{S} ; \forall t \in \mathcal{T}
$$$$
\forall j \in \mathcal{J} ; \forall k \in \mathcal{S} ; \forall t \in \mathcal{T}: t>1(10)
$$

$\forall i \in \mathcal{I} ; \forall j \in \mathcal{J} ; \forall k \in \mathcal{S} ; \forall t \in \mathcal{T}(12)$

$\forall h \in \mathcal{S} ; \forall k \in \mathcal{S} ; \forall t \in \mathcal{T}(13)$

The objective function (1) maximises the total amount of demand served over service types and decision periods. Equation (2) enforces that each facility can have at most one service type to provide. Equation (3) ensures that the demand for service type $k$ cannot be provided from a potential facility at $j \in \mathcal{J}$ unless there is a facility at $j \in \mathcal{J}$ able to provide a service of type $k$ or higher and the satisfied demand cannot exceed the total number of people attracted to the facility. Equation (4) guarantees that the total satisfied demand of demand node $i \in \mathcal{I}$ does not exceed its demand. Equation (5) governs that the total demand assigned to a facility should not exceed its capacity. Equation (6) deals with the budget constraint and ensures that the total spending is below the available budget at each period. Note that since a reduction of service type is not allowed, the values of $e_{j h k}^{t}$ for $k<h$ is set to a sufficiently large number (e.g. infinity). Equations (7)-(9) ensure that variable $z_{j h k}^{t}$ can take a value of one if $x_{j h}^{t-1}$ and $x_{j k}^{t}$ both equal one. Equation (10) ensures that the service type of each facility does not decrease in consecutive periods. In addition, Equations (11)-(13) ensure that $x_{j k}^{t}$ and $z_{j h k}^{t}$ are binary and $y_{i j k}^{t}$ takes positive values.

\section{Solution Method}

Using heuristic methods is a well-established way to solve combinatorial optimisation problems. Various heuristics are developed to solve a variety of applications, such as emergency routing problem (Fontem et al., 2016), districting problem (Rios-Mercado and Escalante, 2016) and scheduling (Liu et al., 2017). Among these methods, Iterated Local Search (ILS) is an efficient and yet conceptually simple local search meta-heuristic algorithm, which was first proposed by Lourenço et al. (2003) as a general solution algorithm and has been used for a variety of facility location problems so far such as Nguyen et al. (2012), de Armas et al. (2018) and Wu et al. (2017). The essence of ILS is based on iteratively searching a small subset of the solution space, using a set of local search operators, following a perturbation operator which ensures enough diversification in the heuristic. A sketch of the iterated local search procedure is given in Algorithm 1. 


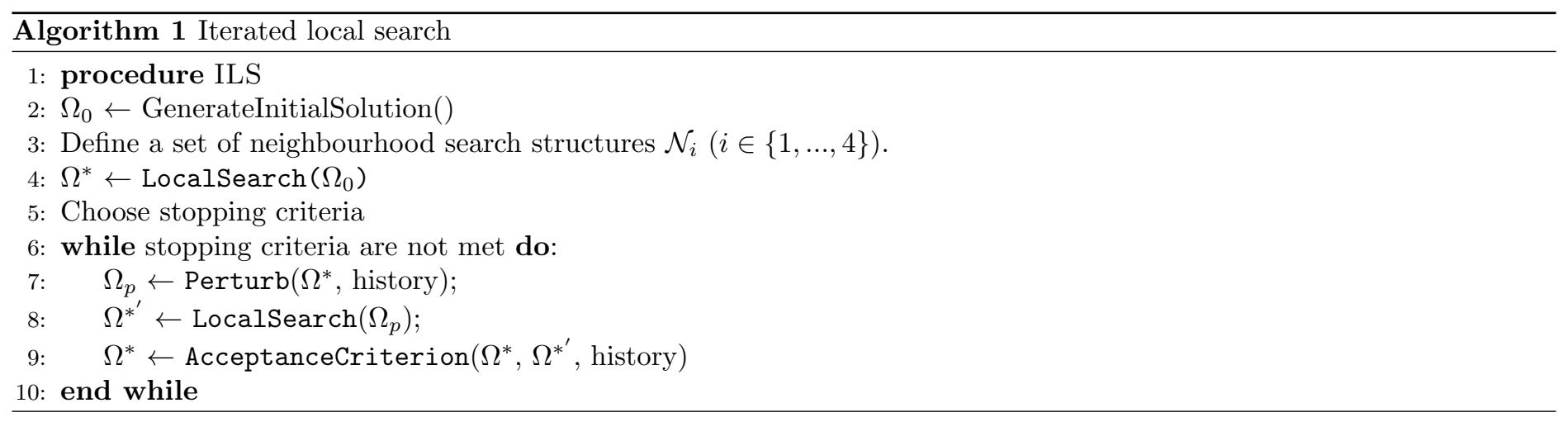

Similar to other meta-heuristic methods, ILS can generate infeasible solutions, and this solution is penalised by adding a penalty term to the objective function value (which is negative in our problem as a maximisation problem). The efficient exploitation of these penalised solutions is known to contribute significantly to the performance of heuristics (Vidal et al., 2013). Hence, we incorporated a long-term memory structure similar to Li et al. (2015) and replaced a solution found in the local search step if it outperformed the incumbent or with a probability of $\frac{1}{\rho \sqrt{\kappa+1}}(\rho$ and $\kappa$ are a parameter and the number of times a worse solution was accepted in previous runs respectively). This enhanced the diversification of the heuristic, changed the search trajectory and led to better solutions. Tuning the $\rho$ parameter is of utmost importance in the success of the proposed heuristic as it determines the search trajectory, and the heuristic's diversification level. While a high value of $\rho$ can lead to the algorithm being trapped in local optima, lower values can make the search random. Tuning the $\rho$ parameter will be explained in Section (5). In the remainder of this section, the proposed solution representation scheme is presented as well as the neighbourhood search structures before numerical experiments are presented.

\subsection{Solution representation}

Given a set $\mathcal{J}=\{1,2, \ldots, n\}$ of facilities and $\mathcal{T}=\left\{1,2, \ldots, l_{\mathcal{T}}\right\}$ periods, a solution to the problem can be represented as a vector of elements $\Omega=\left\{\omega_{11}, \ldots, \omega_{1 l_{\mathcal{T}}}, \ldots, \omega_{n 1}, \omega_{n 2}, \ldots, \omega_{n l_{\mathcal{T}}}\right\}$ where $\omega_{j t}$ shows the service type offered at node $j$ and time $t$. In other words, assuming $n$ facilities, $l_{\mathcal{S}}$ types and $l_{\mathcal{T}}$ periods, each solution can be represented as a vector of size $n \times l_{\mathcal{T}}$ with integer values between 0 and $l_{\mathcal{S}}$ for each of its elements. A value of zero for an element shows that there is no service/facility at that node and period.

Figure 1 demonstrates a sample solution with three potential facilities, four service types and six periods. In this example, while there is no facility at node one in the first three periods, service types one, two and four are offered in periods four, five and six respectively. One should note that in a valid solution and for each potential facility, the service types are nondecreasing by the period. This representation scheme has a number of attractive features such as being concise which saves computer memory and facilitating calculation of the objective function. Note that the allocation of demands to facilities $\left(y_{i j k}^{t}\right)$ can be done using the nearest neighbour allocation followed by a deterministic greedy re-allocation stage to avoid infeasibility.

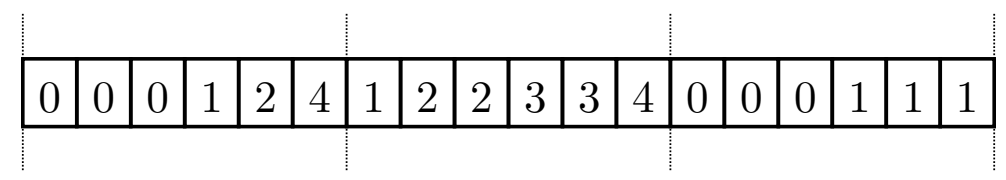

Figure 1: A sample solution representation with $\left(n, l_{\mathcal{S}}, l_{\mathcal{T}}\right)=(3,4,6)$

\subsection{Initialisation Subroutine}

An initial solution is generated using a greedy procedure. To this end, we used a Backward Initialisation procedure to construct the initial solution which starts with locating all the potential facilities and setting their service types at maximum. Then, in each iteration, the service type is reduced by one, following a reverse roulette wheel selection method. In other words, the probability of removing a facility is inversely proportional to the aggregate demand in the network to be captured by the facility. This procedure is repeated until the solution becomes feasible. The pseudo-code of the algorithms is given in Algorithm 2. 


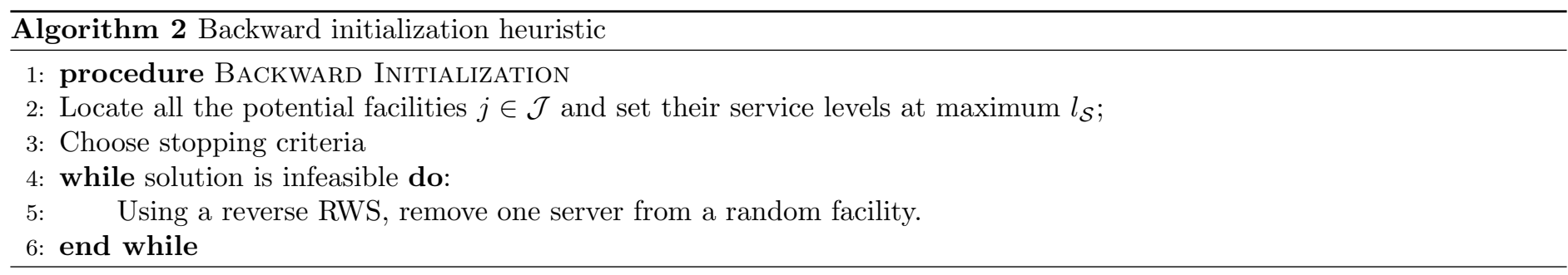

\subsection{Neighbourhood search}

We construct four neighbourhood search structures (moves) to maintain a balance between intensification and diversification of the heuristic. The first move, cross-exchange was used as the perturbation mechanism and the other three acted as local search moves. One should note that in using these local search moves, a best-first strategy has been used. In other words, if a move improved the incumbent solution, it was applied without testing the effect of other local search moves.

\section{Cross-exchange}

To enhance the diversification of the procedure, this mechanism randomly selects two nodes $j_{1} \in \mathcal{J}$ and $j_{2} \in \mathcal{J}$ and exchanges the values associated with them for all the periods. To put it in simple terms, using a cross-exchange operator, the service level of $j_{1}$ in each period is exchanged with the service level of $j_{2}$ in the same period. For instance, the move $\mathcal{N}_{1}$ in Figure 2

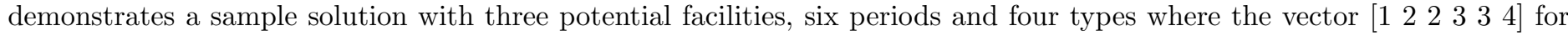
the second facility is swapped with the vector $\left[\begin{array}{llllll}0 & 0 & 0 & 1 & 1 & 1\end{array}\right]$ for the third. This move can lead to violations of budget and capacity which is allowed up to a level and beneficial to enhance the diversification of the algorithm. Overall, using this operator, a maximum of $\frac{2}{n}$ bits of a solution are modified which equals $10 \%$ for a problem with 20 potential facilities. Hence, this move keeps the balance between diversification and intensification by not being too strong to make the search random and not being too weak, so the local search heuristic reverses it.

\section{Service initiation}

The move takes place when a facility is opened and its types are allocated in each period following a non-decreasing order. In other words, the move takes a solution $\Omega=\left\{\omega_{11}, \ldots, \omega_{n l_{\mathcal{T}}}\right\}$, finds a facility which is not operational in any period and assigns random non-decreasing service types to it. The move $\mathcal{N}_{2}$ in Figure 2 demonstrates how the move works for a sample solution where the the first facility has been affected by this move and non-decreasing service types are added to it for periods one to six.

\section{Down-sizing}

This move tries to avoid infeasibility due to budget constraints by iteratively decreasing the service type until the solution becomes feasible (see $\mathcal{N}_{3}$ in Figure 2 as an example). One important issue with this move is to make sure that in the new solution, the non-decreasing order of service types is not violated after carrying out the move. To this end, a random node and period is selected, and its service type is decreased before the service types of the following periods for the same node are adjusted accordingly. This procedure is followed until a feasible solution is obtained.

\section{Up-sizing}

This move corresponds to increasing the service types of an open facility and making sure that service types are in a nondecreasing order. The move $\mathcal{N}_{4}$ in Figure 2 demonstrates the case where this move leads to increasing the service types of facility two for periods one to six. 


$$
\begin{gathered}
\Omega_{1} \\
\Omega_{2}: \mathcal{N}_{1} \text { applied to } \Omega_{1} \\
\Omega_{3}: \mathcal{N}_{2} \text { applied to } \Omega_{2} \\
\Omega_{4}: \mathcal{N}_{3} \text { applied to } \Omega_{3} \\
\Omega_{5}: \mathcal{N}_{4} \text { applied to } \Omega_{4}
\end{gathered}
$$

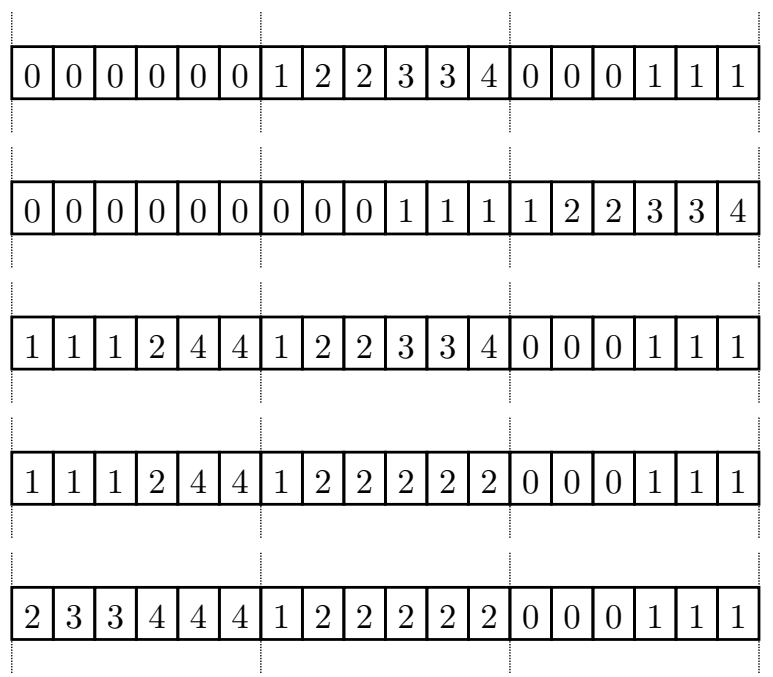

Figure 2: Sequential deployment of neighbourhood search mechanisms

\section{Computational experiments}

In this section, the results of solving a set of randomly generated instances using the Gurobi Optimization (2016) as well as the proposed heuristic is discussed.

\subsection{Test problems}

To the best of our knowledge, there is not standard dataset for MCFLP-BC to test the performance of the proposed heuristic. Hence, we used the following scheme to generate the instances with various settings ${ }^{1}$. We divided the test problems into three subsets, namely small-scale (SS), medium-scale (MS) and large-scale (LS) depending on the number of demand nodes and potential facilities. Initial demands $\left(d_{i k}^{0}\right)$ were generated following a uniform distribution between 100 and 1,000 which is changed by being multiplied by a random number between 0.9 and 1.2 in each period and rounded up to the nearest integer values. This allowed for both negative and positive growth rates in the population of each node which is realistic considering the effect of demographic changes. Given the demands, the capacities $\lambda_{j k}$ and variable costs $g_{j k}^{t}$ were generated following uniform distributions $\mathcal{U}\left(0, \zeta \overline{d_{i k}^{t}}\right)$ and $\mathcal{U}\left(0,0.1 \overline{d_{i k}^{t}}\right)$ where $\overline{d_{i k}^{t}}$ is the average demand over periods and $\zeta$ is a given parameter. We solved the capacitated $(\zeta=1)$ and uncapacitated $(\zeta=\infty)$ versions of the problem to see the effect of capacity constraints on the solution quality and runtime. In order to generate the coordinates for demand nodes and potential facilities, we generated problems with symmetric (uniform, normal and multi-modal) and skewed (Beta) distributions with coordinates in the range $[0,100]$ as shown in Figure 3 which mimics the demand distribution in the real-world. For instance, while the population of Australia and China are skewed to east, the population of Turkey and Poland are almost uniformly distributed across the country and the population of the United States is scattered following a multi-modal distribution (the same patterns exist for cities such as London, Tokyo and Sao Paolo). We then use the Euclidean distance to find the distance between nodes. We generated the budgets $b^{t}$ of each period between a lower limit and an upper limit with changes following one of the four variants which are given in Figure 4. One should note that we set an identical range for the budgets in the test problems to have a fair comparison between budget types. Finally, the fixed $\left(f_{j}^{t}\right)$ and the service type change $\left(e_{j h k}^{t}\right)$ costs were generated based on uniform distributions $\mathcal{U}\left(b_{\max } / 5 ; b_{\max }\right)$ and $\mathcal{U}\left(0 ; b_{\max } / 3\right)$ respectively where $b_{\max }$ is the maximum budget generated over different periods.

Note that in generating the test problems, the values of $b^{t}$ and $\lambda_{j t}$ were guaranteed to be higher than a certain threshold, thereby ensuring presence of at least one feasible solution. Overall, for each class of test problems (SS, MS and LS), $2 \times 2 \times 2 \times 2 \times 4 \times 4=256$ instances are generated with a total of 768 test problems to solve. Table 1 provides a summary of the test problems and their settings.

\footnotetext{
${ }^{1}$ Test problems are available from the corresponding author upon request.
} 

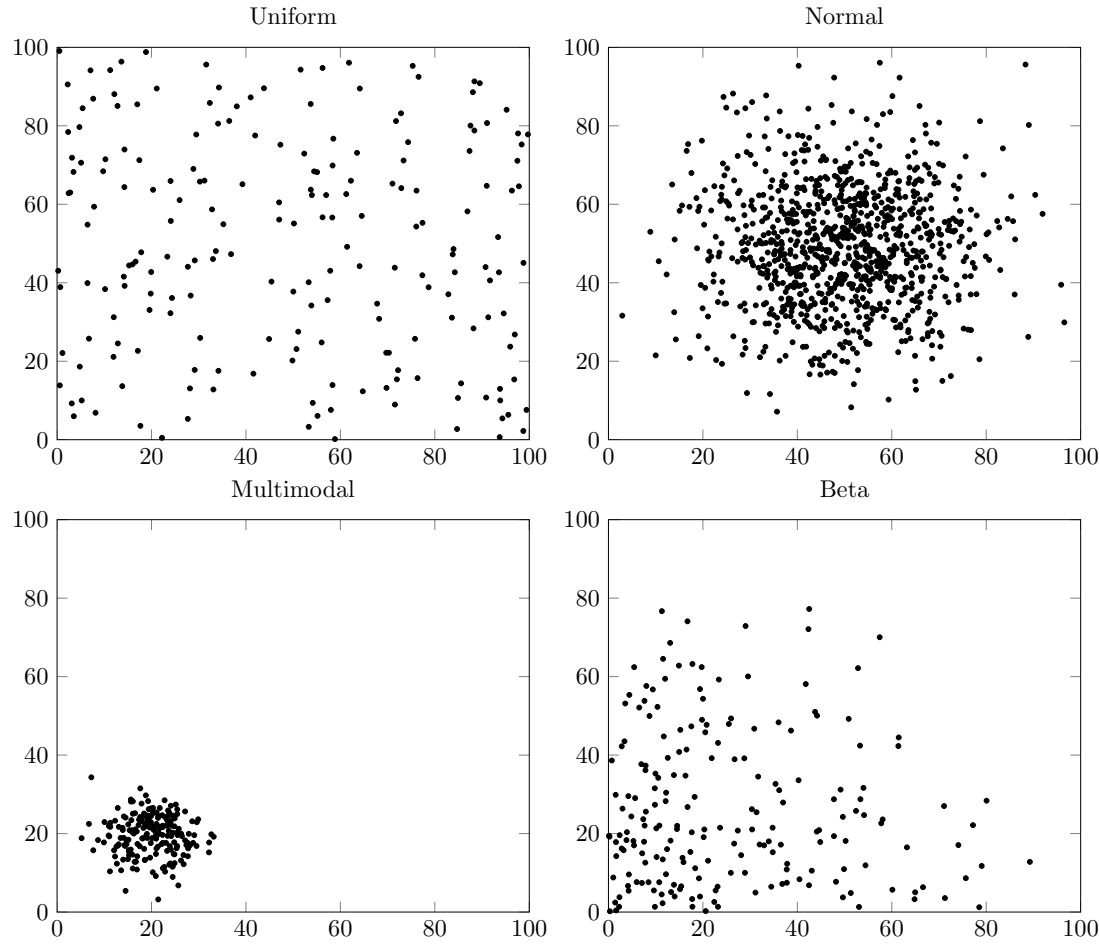

Figure 3: The four types of demand distribution

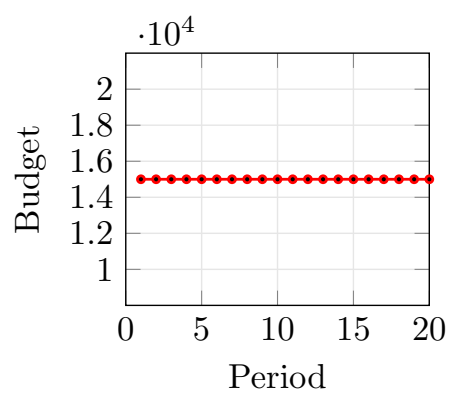

(a)

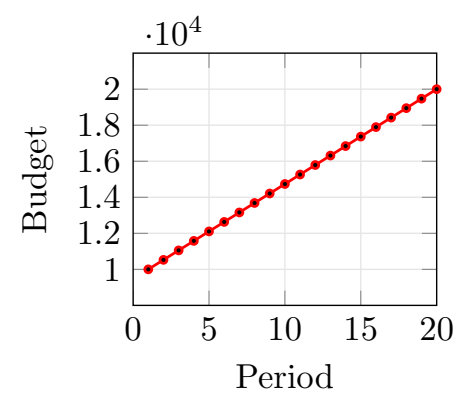

(b)

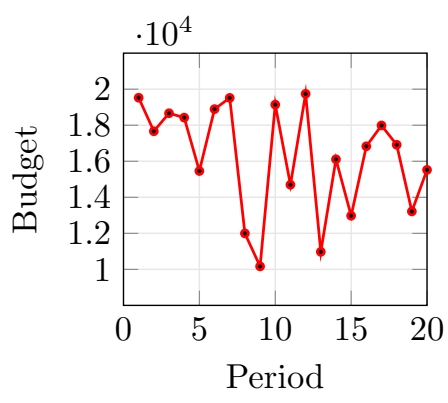

(c)

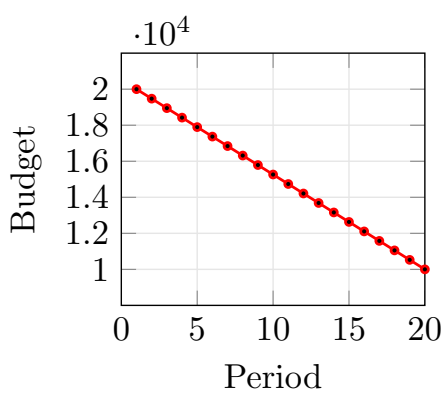

(d)

Figure 4: Four types of budget allocation: (a) constant, (b) increasing, (c) oscillating and (d) decreasing

Table 1: Problem settings

\begin{tabular}{llllllll}
\hline Class & $|\mathcal{I}|$ & $|\mathcal{J}|$ & $|\mathcal{S}|$ & $|\mathcal{T}|$ & $\zeta$ & Distribution & Budget type \\
\hline Small-scale & 10 & $\{5,10\}$ & $\{3,6\}$ & $\{4,8\}$ & $\{1, \infty\}$ & Uniform, Beta, Multi-modal, Normal & Inc/Dec/Con/Osc \\
Medium-scale & 20 & $\{5,10\}$ & $\{3,6\}$ & $\{4,8\}$ & $\{1, \infty\}$ & Uniform, Beta, Multi-modal, Normal & Inc/Dec/Con/Osc \\
Large-scale & 50 & $\{10,20\}$ & $\{5,10\}$ & $\{5,10\}$ & $\{1, \infty\}$ & Uniform, Beta, Multi-modal, Normal & Inc/Dec/Con/Osc \\
\hline
\end{tabular}

\subsection{Numerical results}

In this section, the performance of the proposed solution procedure is presented by means of computational experiments on a variety of problem settings as given in Section 5.1. The mathematical model was implemented in Python using Gurobi Optimizer 8.0.0 (Gurobi Optimization, 2016) and the heuristic was compiled and executed in Python on a 64-bit Core i5 laptop with 16 GBytes of memory. We imposed a time limit of two hours (7,200 seconds) for Gurobi and ran the heuristic for $m l_{\mathcal{T}}$ seconds before evaluating the performance of the proposed ILS with respect to both the solution quality and runtime. In choosing a termination criterion, we tested the performance of a number of options (such as an arbitrary number of iterations) 
for our problem and used an adaptive termination criterion (as mentioned above) for our heuristic given its promising results, the fact that improvements are observed throughout the runs for the vast majority of the instances and that there are hardly any improvements after this time limit.

Similar to other heuristics, parameter calibration is important for the proposed solution procedure. To choose the best value for $\rho$, we investigated the performance of the proposed ILS with values of $\rho \in\{1,2, \ldots, 10\}$. Then, we performed an ANOVA test and generated the Tukey intervals with $95 \%$ confidence level to see if the difference in results were statistically significant. The results of these runs showed that the algorithm is sensitive to the choice of $\rho$ and choosing a value of $\rho=3$ can lead to the best results. Hence, we used a value of $\rho=3$ in our numerical experiments.

Running the experiments suggested that similar to many other combinatorial optimisation problems, the CPU time to solve instances increase dramatically after a certain point and this runtime is more sensitive to an increase in the number of periods rather than any other parameter. On average, the exact algorithm needs more time for higher values of $\eta$ and the capacitated version $(\zeta=1)$ which are both intuitive. In order to examine the performance of the proposed ILS, we used the Relative Percentage Deviation (RPD) measure shown as $\Delta$ and found using Equation (14):

$$
\Delta=\frac{\Psi_{G}-\Psi_{H}}{\Psi_{G}}
$$

where $\Psi_{G}$ and $\Psi_{H}$ are the best-found solutions using the exact procedure and the proposed ILS respectively. It is remarkable that $\Psi_{G}$ equals the optimal solution in case Gurobi found the optimal solution within the time limit of two hours and to the best bound if it failed to reach an optimal within the time limit. We compared the performance of the proposed ILS with the results of Gurobi for the test problems and concluded that the proposed heuristic outperforms Gurobi in terms of runtime, while the quality of it solutions are comparable with exact ones (average gaps of $0.02 \%, 0.16 \%$ and $0.76 \%$ for the three classes of test problems). Moreover, its gaps do not exceed $1.4 \%$ for those cases with a known optimal solution while it needs considerably less time compared to an exact algorithm. The results also confirm that the proposed heuristic reached the optimal solution in 213 out of the 768 instances which equals $27.7 \%$ of problem instances. Figure 5 demonstrates the gap of the proposed ILS for test problems with different values of $(m, n)$. As expected, the gaps for larger instances are higher; however, the average gap for the instances is below $1 \%$ for all the six combinations of $(m, n)$. To gain some insights into the effect of geographical distribution of nodes on the performance of the proposed ILS, we did an additional comparison as given in Figure 6. Results show that while on average, our proposed ILS performs worse for normal distribution of nodes, the proposed ILS performs almost the same for beta and multi-modal distributions.

To look into the problem more, we carried out some experiments using the proposed heuristic to examine how robust the results of the heuristic are. To this end, we ran our heuristic on a set of test problems with $m \in\{60,80\}, n \in\{30,40\}$, $l_{\mathcal{S}}=20, l_{\mathcal{T}}=20, \zeta=1$ and 16 combinations of demand distribution and budget allocation with ten runs each. The results of these numerical experiments are given in Table (2) where $\mu, \sigma$, and C.V represent the average, standard deviation and coefficient of variation of the runs. Please note that the Opt-Gap columns report the optimality gap in Gurobi after 7,200 seconds for each problem setting. Results show that the proposed heuristic is robust in terms of its outputs with coefficients of variation between $0.58 \%$ and $4.84 \%$.

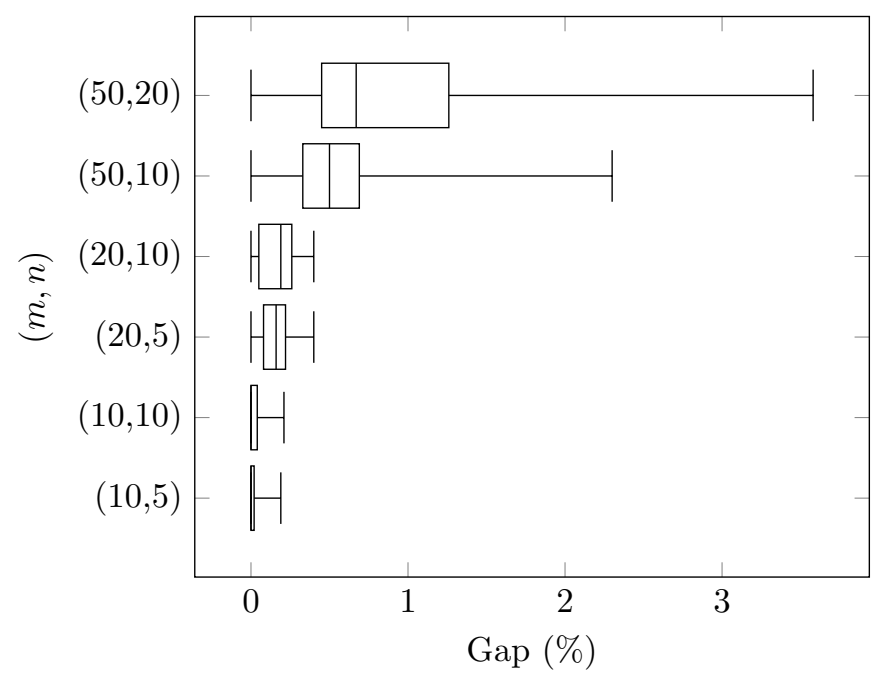

Figure 5: Box-whisker diagrams of errors for combinations of $(m, n)$ 


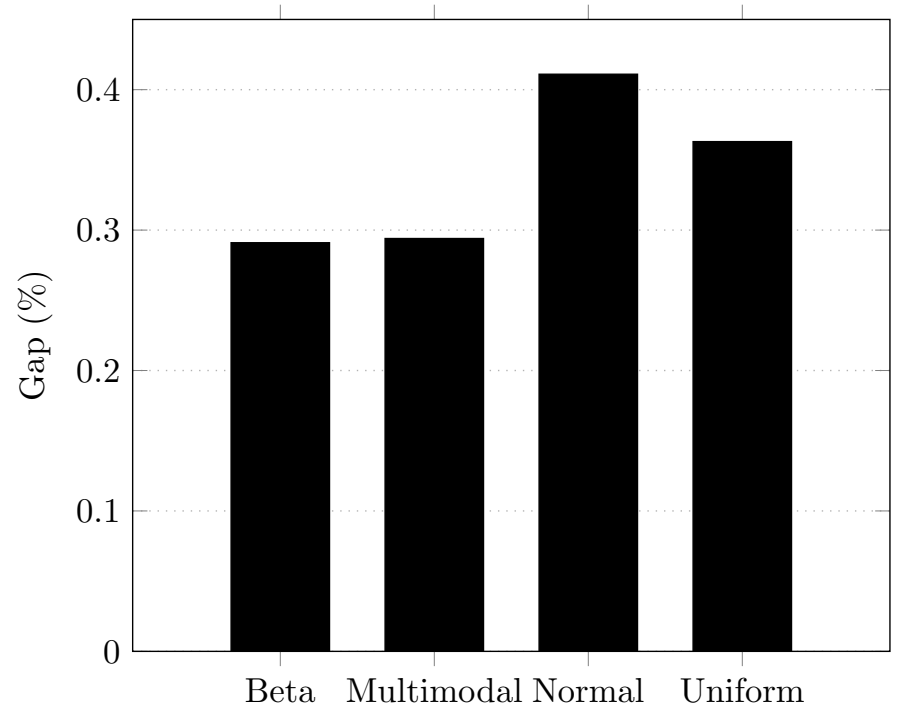

Figure 6: Comparing the performance of the proposed procedure for geographical distributions

\section{Managerial insights}

In order to gain insight into the sensitivity of the problem to changes in problem settings, we analysed the results of the exact algorithm and compared solutions with different settings using a Scheffé test at five percent of significance. The results clearly showed that while the objective values are relatively higher for the constant allocation of budget, an increasing allocation of budget leads to the lowest coverage of demand. Considering that the minimum and maximum budget levels are identical for the four budget schemes and our assumption of consistent services, this observation can be justified for data sets like ours where the population changes are not dramatic between two consecutive periods. Another observation with different problem settings is that while a normal distribution of demand leads to the highest coverage of demand under budget and capacity constraints, the uniform distribution leads to the lowest. This observation is intuitive since covering a set of demand points with a uniform distribution needs more facilities compared to the case with a normal distribution of demand points. This observation is valid if capacities are large enough and if the value of $\eta$ is not too small to make the facility attractive to demand nodes regardless of their distance to travel.

To examine the effect of continuous service, we ran the model for the inconsistent version and compared the results with the consistent one. To this end and to model the inconsistent version, constraint 10 was relaxed and two new binary variables were introduced to the model as following.

$$
\begin{aligned}
& u_{j k t}^{o}=\left[\sum_{k \in \mathcal{S}} x_{j k}^{t}-\sum_{k \in \mathcal{S}} x_{j k}^{t-1}\right]^{+}=\max \left\{0, \sum_{k \in \mathcal{S}} x_{j k}^{t}-\sum_{k \in \mathcal{S}} x_{j k}^{t-1}\right\} \\
& u_{j k t}^{c}=\left[\sum_{k \in \mathcal{S}} x_{j k}^{t-1}-\sum_{k \in \mathcal{S}} x_{j k}^{t}\right]^{+}=\max \left\{0, \sum_{k \in \mathcal{S}} x_{j k}^{t-1}-\sum_{k \in \mathcal{S}} x_{j k}^{t}\right\}
\end{aligned}
$$

and the following constraints were added to the model to keep the model linear. Please note that there is no need to add a constraint on $u_{j k t}^{c}$ and $u_{j k t}^{o}$ to be positive as they will be added as binary variables to the model.

$$
\begin{aligned}
& u_{j k t}^{o} \geq \sum_{k \in \mathcal{S}} x_{j k}^{t}-\sum_{k \in \mathcal{S}} x_{j k}^{t-1} \\
& u_{j k t}^{c} \geq \sum_{k \in \mathcal{S}} x_{j k}^{t-1}-\sum_{k \in \mathcal{S}} x_{j k}^{t} \\
& u_{j k 1}^{o}=x_{j k}^{1} \\
& u_{j k 1}^{c}=0
\end{aligned}
$$

$$
\begin{array}{r}
\forall j \in \mathcal{J} ; t \in \mathcal{T}: t>1 \\
\forall j \in \mathcal{J} ; t \in \mathcal{T}: t>1 \\
\forall j \in \mathcal{J} ; k \in \mathcal{S} \\
\forall j \in \mathcal{J} ; k \in \mathcal{S}
\end{array}
$$

Then, the inconsistent version of the problem (MIFLP-BC) is modelled as following with Constraints (2)-(5) and (7)-(9) 
and (11)-(13):

$$
\begin{array}{lr}
\text { (MIFLP-BC): } \max \sum_{i \in \mathcal{I}} \sum_{j \in \mathcal{J}} \sum_{k \in \mathcal{S}} \sum_{a t \in \mathcal{T}} y_{i j k}^{t} & \\
\sum_{j \in \mathcal{J}} \sum_{k \in \mathcal{S}} f_{j t}^{o} u_{j k t}^{o}+\sum_{j \in \mathcal{J}} \sum_{k \in \mathcal{S}} f_{j t}^{c} u_{j k t}^{c}+\sum_{j \in \mathcal{J}} \sum_{h \in \mathcal{S}} \sum_{k \in \mathcal{S}} e_{j h k}^{t} z_{j h k}^{t}+\sum_{i \in \mathcal{I}} \sum_{j \in \mathcal{J}} \sum_{k \in \mathcal{S}} g_{j k}^{t} y_{i j k}^{t} \leq b^{t} & \forall t \in \mathcal{T} \\
u_{j k t}^{o} \geq \sum_{k \in \mathcal{S}} x_{j k}^{t}-\sum_{k \in \mathcal{S}} x_{j k}^{t-1} & \forall j \in \mathcal{J} ; t \in \mathcal{T}: t>1 \\
u_{j k t}^{c} \geq \sum_{k \in \mathcal{S}} x_{j k}^{t-1}-\sum_{k \in \mathcal{S}} x_{j k}^{t} & \forall j \in \mathcal{J} ; t \in \mathcal{T}: t>1 \\
u_{j k 1}^{o}=x_{j k}^{1} & \forall j \in \mathcal{J} ; k \in \mathcal{S} \\
u_{j k 1}^{c}=0 & \forall j \in \mathcal{J} ; k \in \mathcal{S}
\end{array}
$$

We designed a set of four test problems with 40 demand nodes, six periods and three service levels and assumed that each demand node can host a facility and that $f_{j t}^{c}=0.5 \times f_{j t}^{o}$. We then compared the results of the consistent version against the inconsistent version for different distribution of parameters as given in Figure 7 where circles represent demand nodes, and triangles show the nodes which host a facility in at least one of the periods. Results showed that for the inconsistent version, the number of nodes hosting a facility can be up to three times the number of facilities in the consistent version (It should be noted that these results are sensitive to the values of $f_{j t}^{o}$ and $f_{j t}^{c}$ ). It is worth saying that although the coverage levels in the case of inconsistent services is never below the case of consistent services, an inconsistent service is not possible in some cases due to a variety of reasons such as regulations set by the governments or the exorbitant costs for a reorganisation of the network. All in all, if opening and closure of facilities in different periods is feasible, the inconsistent version of the problem always leads to at least equally good solutions in terms of demand coverage. 

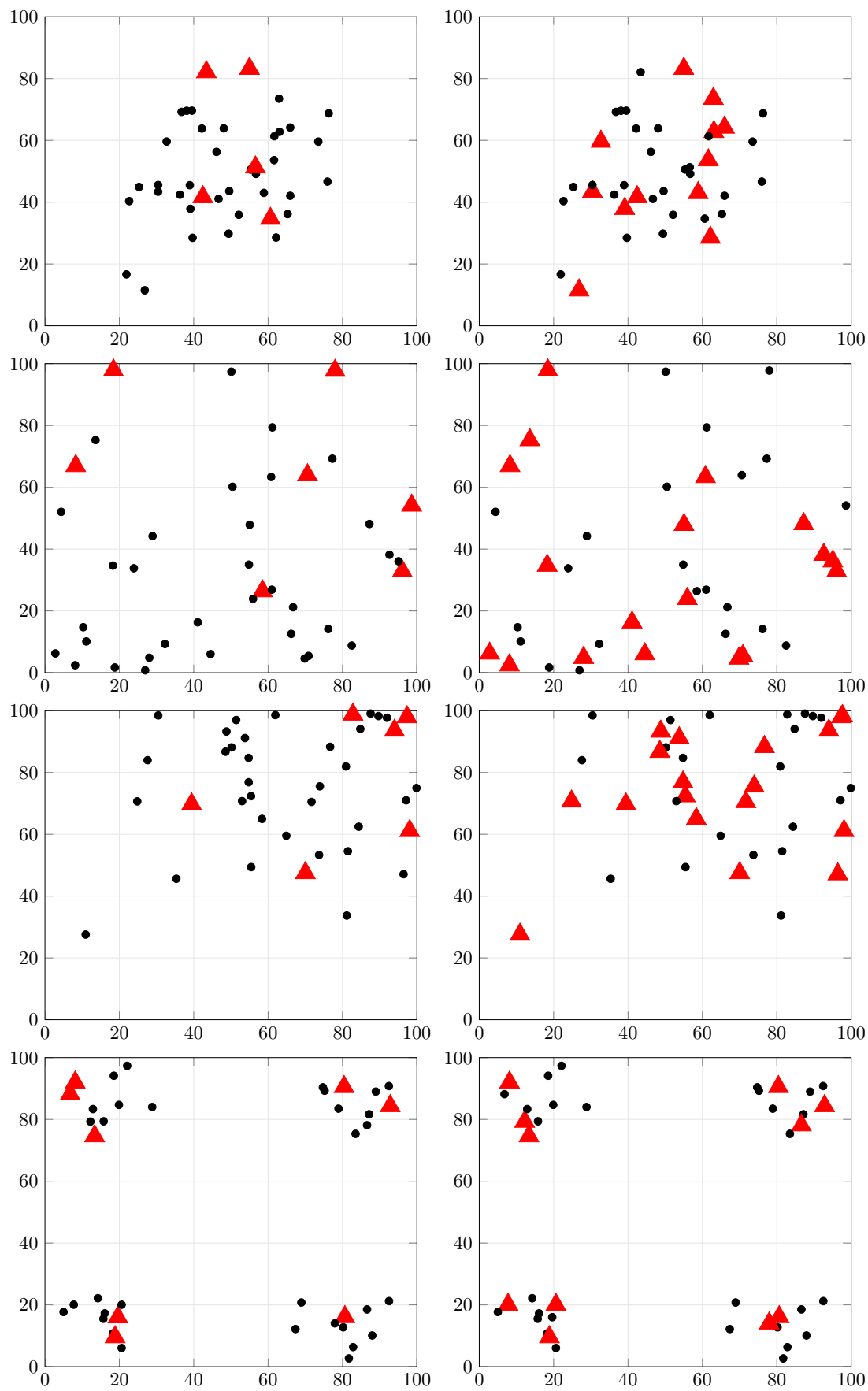

Figure 7: Results of the consistent (left) vs inconsistent (right) versions for normal, uniform, beta and multi-modal distribution of nodes (top to bottom)

Policymakers can make use of this model in designing the network or alternatively comparing the network's status-quo with the optimal setting. Additionally, the model can be easily adapted to import the current setting and to plan forward. One advantage of the model is that it enables decision-makers to adjust decisions over a planning horizon. In other words, decision makers can adapt a rolling horizon approach in which the planning horizon is rolled over after implementing the results for each period, enabling decision makers to incrementally build the network while considering the evolution of parameters.

\section{Conclusions and future research}

In this paper, a multi-period facility location problem was studied to locate elderly centres with budget and capacity considerations. We developed a novel mixed integer programming formulation for the problem and proposed an efficient 
iterated local search procedure to solve it. The performance of the heuristic was tested on a set of 768 test problems with different settings. The computational results showed that the proposed algorithm can provide near-optimal solutions with average gaps of $0.02 \%, 0.16 \%$ and $0.76 \%$ for small-scale, medium-scale and large-scale problems respectively in less time than the exact solutions.

Further research on this topic may include addition of uncertainty to the model and solving the robust version of the problem. Moreover, to make the model more realistic, further research should be undertaken to investigate the effect of incorporating the elderlies' behaviours in modelling the attractiveness of the facilities. This can be achieved through undertaking primary research to extract the factors affecting the elderlies' choice. Besides, one can investigate the performance of some other heuristics to solve the problem with more complicated settings. We believe that although the our research is not a perfect presentation of the real problem, it adds to the growing body of literature on community operations research. We suggest further research on the topic using real-world data as another future research area for interested researchers. This should be done in consultation with practitioners in the field of elderly care and by informing the model with further constraints which can be specific to a region and its culture.

Table 2: Results of running the heuristic with $m \in\{60,80\}$

\begin{tabular}{|c|c|c|c|c|c|c|c|c|c|c|c|c|c|}
\hline \multirow{2}{*}{$n$} & \multirow{2}{*}{$l_{\mathcal{S}}$} & \multirow{2}{*}{$l_{\mathcal{T}}$} & \multirow{2}{*}{$\lambda$} & \multirow{2}{*}{ Distribution } & \multirow{2}{*}{ Budget } & \multicolumn{4}{|l|}{$m=60$} & \multicolumn{4}{|l|}{$m=80$} \\
\hline & & & & & & Opt-Gap & $\mu$ & $\sigma$ & $\mathrm{CV}$ & Opt-Gap & $\mu$ & $\sigma$ & $\mathrm{CV}$ \\
\hline 30 & 20 & 20 & 1 & Beta & Con & $72.83 \%$ & 127,429 & 4,443 & $3.49 \%$ & $70.98 \%$ & 134,587 & 2,545 & $1.89 \%$ \\
\hline 30 & 20 & 20 & 1 & Beta & Dec & $47.65 \%$ & 144,790 & 2,718 & $1.88 \%$ & $78.78 \%$ & 154,351 & 4,924 & $3.19 \%$ \\
\hline 30 & 20 & 20 & 1 & Beta & Osc & $130.57 \%$ & 135,933 & 3,310 & $2.44 \%$ & $115.73 \%$ & 143,599 & 4,958 & $3.45 \%$ \\
\hline 30 & 20 & 20 & 1 & Beta & Inc & $15.59 \%$ & 127,734 & 3,271 & $2.56 \%$ & $22.62 \%$ & 135,360 & 1,924 & $1.42 \%$ \\
\hline 30 & 20 & 20 & 1 & Multimodal & Con & $66.46 \%$ & 122,849 & 1,122 & $0.91 \%$ & $67.06 \%$ & 129,044 & 5,913 & $4.58 \%$ \\
\hline 30 & 20 & 20 & 1 & Multimodal & Dec & $91.52 \%$ & 149,402 & 2,128 & $1.42 \%$ & 108.43 & 159,775 & 4,017 & $2.51 \%$ \\
\hline 30 & 20 & 20 & 1 & Multimodal & Osc & $113.22 \%$ & 138,355 & 3,693 & $2.67 \%$ & $85.07 \%$ & 145,040 & 6,655 & $4.59 \%$ \\
\hline 30 & 20 & 20 & 1 & Multimodal & Inc & $15.74 \%$ & 127,153 & 3,244 & $2.55 \%$ & $23.55 \%$ & 134,534 & 5,949 & $4.42 \%$ \\
\hline 30 & 20 & 20 & 1 & Normal & Con & $29.31 \%$ & 126,062 & 1,098 & $0.87 \%$ & $49.75 \%$ & 133,773 & 1,819 & $1.36 \%$ \\
\hline 30 & 20 & 20 & 1 & Normal & Dec & $103.97 \%$ & 143,866 & 1,937 & $1.35 \%$ & $70.05 \%$ & 151,067 & 5,383 & $3.56 \%$ \\
\hline 30 & 20 & 20 & 1 & Normal & Osc & $122.03 \%$ & 131,435 & 2,242 & $1.71 \%$ & $113.73 \%$ & 138,193 & 3,495 & $2.53 \%$ \\
\hline 30 & 20 & 20 & 1 & Normal & Inc & $15.43 \%$ & 138,610 & 1,439 & $1.04 \%$ & $17.65 \%$ & 142,314 & 1,150 & $0.81 \%$ \\
\hline 30 & 20 & 20 & 1 & Uniform & Con & $66.57 \%$ & 122,296 & 1,992 & $1.63 \%$ & $61.45 \%$ & 128,191 & 1,404 & $1.10 \%$ \\
\hline 30 & 20 & 20 & 1 & Uniform & Dec & $40.60 \%$ & 150,153 & 3,117 & $2.08 \%$ & $84.58 \%$ & 155,870 & 3,808 & $2.44 \%$ \\
\hline 30 & 20 & 20 & 1 & Uniform & Osc & $112.55 \%$ & 146,594 & 4,802 & $3.28 \%$ & $121.04 \%$ & 154,697 & 5,217 & $3.37 \%$ \\
\hline 30 & 20 & 20 & 1 & Uniform & Inc & $22.81 \%$ & 130,340 & 1,671 & $1.28 \%$ & $17.29 \%$ & 137,587 & 2,143 & $1.56 \%$ \\
\hline 40 & 20 & 20 & 1 & Beta & Con & $101.21 \%$ & 152,421 & 2,504 & $1.64 \%$ & $293.67 \%$ & 161,867 & 6,071 & $3.75 \%$ \\
\hline 40 & 20 & 20 & 1 & Beta & Dec & $116.88 \%$ & 173,187 & 5,343 & $3.08 \%$ & $118.80 \%$ & 180,710 & 2,424 & $1.34 \%$ \\
\hline 40 & 20 & 20 & 1 & Beta & Osc & $127.63 \%$ & 162,593 & 2,671 & $1.64 \%$ & $147.22 \%$ & 170,676 & 3,152 & $1.85 \%$ \\
\hline 40 & 20 & 20 & 1 & Beta & Inc & $30.96 \%$ & 152,785 & 2,069 & $1.35 \%$ & $33.31 \%$ & 156,472 & 2,804 & $1.79 \%$ \\
\hline 40 & 20 & 20 & 1 & Multimodal & Con & $105.98 \%$ & 146,942 & 3,698 & $2.52 \%$ & $302.41 \%$ & 156,534 & 913 & $0.58 \%$ \\
\hline 40 & 20 & 20 & 1 & Multimodal & Dec & $140.46 \%$ & 178,704 & 2,889 & $1.62 \%$ & $130.15 \%$ & 185,769 & 4,812 & $2.59 \%$ \\
\hline 40 & 20 & 20 & 1 & Multimodal & Osc & $107.60 \%$ & 165,490 & 2,699 & $1.63 \%$ & $83.47 \%$ & 171,106 & 1,120 & $0.65 \%$ \\
\hline 40 & 20 & 20 & 1 & Multimodal & Inc & $36.31 \%$ & 152,091 & 4,714 & $3.10 \%$ & $27.39 \%$ & 157,716 & 7,633 & $4.84 \%$ \\
\hline 40 & 20 & 20 & 1 & Normal & Con & $91.47 \%$ & 150,786 & 3,051 & $2.02 \%$ & $82.21 \%$ & 159,753 & 1,452 & $0.91 \%$ \\
\hline 40 & 20 & 20 & 1 & Normal & Dec & $126.63 \%$ & 172,082 & 1,231 & $0.72 \%$ & $512.27 \%$ & 180,325 & 2,178 & $1.21 \%$ \\
\hline 40 & 20 & 20 & 1 & Normal & Osc & $155.12 \%$ & 157,213 & 1,472 & $0.94 \%$ & $173.17 \%$ & 166,081 & 6,488 & $3.91 \%$ \\
\hline 40 & 20 & 20 & 1 & Normal & Inc & $38.39 \%$ & 165,794 & 4,345 & $2.62 \%$ & $153.27 \%$ & 173,617 & 3,976 & $2.29 \%$ \\
\hline 40 & 20 & 20 & 1 & Uniform & Con & $96.00 \%$ & 146,281 & 3,884 & $2.65 \%$ & $303.15 \%$ & 156,215 & 2,654 & $1.70 \%$ \\
\hline 40 & 20 & 20 & 1 & Uniform & Dec & $129.57 \%$ & 179,602 & 1,579 & $0.88 \%$ & $136.02 \%$ & 186,689 & 1,429 & $0.77 \%$ \\
\hline 40 & 20 & 20 & 1 & Uniform & Osc & $132.24 \%$ & 175,345 & 3,601 & $2.05 \%$ & $132.49 \%$ & 183,182 & 2,555 & $1.39 \%$ \\
\hline 40 & 20 & 20 & 1 & Uniform & Inc & $47.72 \%$ & 155,903 & 4,447 & $2.85 \%$ & $39.94 \%$ & 156,102 & 2,171 & $1.39 \%$ \\
\hline
\end{tabular}

\section{References}

Albareda-Sambola, M., Fernández, E., Hinojosa, Y. and Puerto, J. (2009), 'The multi-period incremental service facility location problem', Computers and Operations Research 36(5), 1356-1375. 
Baron, R. C., Rimer, B. K., Breslow, R. A., Coates, R. J., Kerner, J., Melillo, S., Habarta, N., Kalra, G. P., Chattopadhyay, S., Wilson, K. M. et al. (2008), 'Client-directed interventions to increase community demand for breast, cervical, and colorectal cancer screening: a systematic review', American Journal of Preventive Medicine 35(1), S34-S55.

Barros, A. I., Dekker, R. and Scholten, V. (1998), 'A two-level network for recycling sand: a case study', European Journal of Operational Research 110(2), 199-214.

Başar, A., Çatay, B. and Ünlüyurt, T. (2011), 'A multi-period double coverage approach for locating the emergency medical service stations in Istanbul', Journal of the Operational Research Society 62(4), 627-637.

Chan, Y., Mahan, J. M., Chrissis, J. W., Drake, D. A. and Wang, D. (2008), 'Hierarchical maximal-coverage locationallocation: case of generalized search-and-rescue', Computers \& Operations Research 35(6), 1886-1904.

Chung, S. H. and Kwon, C. (2015), 'Multi-period planning for electric car charging station locations: A case of Korean expressways', European Journal of Operational Research 242(2), 677-687.

Currie, J. and Reagan, P. B. (2003), 'Distance to hospital and children's use of preventive care: Is being closer better, and for whom?', Economic Inquiry 41(3), 378-391.

Daskin, M. S., Hesse, S. M. and Revelle, C. S. (1997), ' $\alpha$-reliable $p$-minimax regret: A new model for strategic facility location modeling', Location Science 5(4), 227 - 246.

Davari, S. (2018), 'The incremental cooperative design of preventive healthcare networks', Annals of Operations Research pp. $1-48$.

de Armas, J., Juan, A. and Marques, J. (2018), 'A biased-randomized algorithm for the uncapacitated facility location problem', Advances in Intelligent Systems and Computing 730, 287-298.

Drezner, T. (2014), 'A review of competitive facility location in the plane', Logistics Research 7(1), 1-12.

Drezner, Z. (1995), 'Dynamic facility location: The progressive p-median problem', Location Science 3(1), 1-7.

Duhamel, C., Santos, A. C., Brasil, D., Châtelet, E. and Birregah, B. (2016), 'Connecting a population dynamic model with a multi-period location-allocation problem for post-disaster relief operations', Annals of Operations Research pp. 1-21.

Facione, N. C. (1999), Breast cancer screening in relation to access to health services., in 'Oncology Nursing Forum', Vol. 26, pp. 689-696.

Fazel Zarandi, M., Davari, S. and Haddad Sisakht, S. (2011), 'The large scale maximal covering location problem', Scientia Iranica 18(6), 1564-1570.

Fontem, B., Melouk, S. H., Keskin, B. B. and Bajwa, N. (2016), 'A decomposition-based heuristic for stochastic emergency routing problems', Expert Systems with Applications 59, 47-59.

Franklin, B. (2015), 'What are the economic implications of ageing populations?', https://www.weforum.org/agenda/2015/10/what-are-the-economic-implications-of-ageing-populations/. Online; accessed 14-March-2018.

Galli, L., Letchford, A. and Miller, S. (2018), 'New valid inequalities and facets for the simple plant location problem', European Journal of Operational Research 269(3), 824-833.

Ghahremani-Nahr, J., Kian, R. and Sabet, E. (2019), 'A robust fuzzy mathematical programming model for the closed-loop supply chain network design and a whale optimization solution algorithm', Expert Systems with Applications 116, 454-471.

Government Office for Science (2016), 'Future of an ageing population', https://tinyurl.com/y9qy3s8x. Online; accessed 30-August-2018.

Gurobi Optimization, I. (2016), 'Gurobi Optimizer Reference Manual'.

URL: http://www.gurobi.com

Hinojosa, Y., Kalcsics, J., Nickel, S., Puerto, J. and Velten, S. (2008), 'Dynamic supply chain design with inventory', Computers 83 Operations Research 35(2), 373-391.

Holt-Lunstad, J., Smith, T. B., Baker, M., Harris, T. and Stephenson, D. (2015), 'Loneliness and social isolation as risk factors for mortality: a meta-analytic review', Perspectives on Psychological Science 10(2), 227-237. 
Huff, D. L. (1963), 'A probabilistic analysis of shopping center trade areas', Land Economics pp. 81-90.

Jang, H., Hwang, K., Lee, T. and Lee, T. (2019), 'Designing robust rollout plan for better rural perinatal care system in korea', European Journal of Operational Research 274(2), 730-742.

Johnson, M. P., Gorr, W. L. and Roehrig, S. (2005), 'Location of service facilities for the elderly', Annals of Operations Research 136(1), 329-349.

Johnson, M. P., Midgley, G. and Chichirau, G. (2018), 'Emerging trends and new frontiers in community operational research', European Journal of Operational Research 268(3), 1178-1191.

Knight, V. A., Harper, P. R. and Smith, L. (2012), 'Ambulance allocation for maximal survival with heterogeneous outcome measures', Omega 40(6), 918-926.

Leeson, G., Nanitashvili, N. and Zaloznik, M. (2016), 'Future of an ageing population'.

Li, J., Pardalos, P. M., Sun, H., Pei, J. and Zhang, Y. (2015), 'Iterated local search embedded adaptive neighborhood selection approach for the multi-depot vehicle routing problem with simultaneous deliveries and pickups', Expert Systems with Applications 42(7), 3551-3561.

Lin, F., Kong, N. and Lawley, M. (2012), Capacity planning for publicly funded community based long-term care services, in 'Community-based Operations Research', Springer, pp. 297-315.

Liu, W., Jin, Y. and Price, M. (2017), 'A new improved NEH heuristic for permutation flowshop scheduling problems', International Journal of Production Economics 193, 21-30.

Lourenço, H. R., Martin, O. C. and Stützle, T. (2003), Iterated local search, in 'Handbook of metaheuristics', Springer, pp. $320-353$.

Marianov, V., Eiselt, H. and Luer-Villagra, A. (2018), 'Effects of multipurpose shopping trips on retail store location in a duopoly', European Journal of Operational Research 269(2), 782-792.

Marín, A., Martínez-Merino, L. I., Rodríguez-Chía, A. M. and Saldanha-da Gama, F. (2018), 'Multi-period stochastic covering location problems: modeling framework and solution approach', European Journal of Operational Research 268(2), 432449.

Melo, M. T., Nickel, S. and Saldanha-da Gama, F. (2009), 'Facility location and supply chain management-a review', European Journal of Operational Research 196(2), 401-412.

Mogale, D., Kumar, M., Kumar, S. K. and Tiwari, M. K. (2018), 'Grain silo location-allocation problem with dwell time for optimization of food grain supply chain network', Transportation Research Part E: Logistics and Transportation Review 111, 40-69.

Nguyen, V.-P., Prins, C. and Prodhon, C. (2012), 'A multi-start iterated local search with tabu list and path relinking for the two-echelon location-routing problem', Engineering Applications of Artificial Intelligence 25(1), 56-71.

ONS (2017), 'Overview of the uk population: July 2017', https://tinyurl.com/yc8scczl. Online; accessed 21-June-2018.

Ou-Yang, C. and Ansari, R. (2017), 'Applying a hybrid particle swarm optimization - tabu search algorithm to a facility location case in Jakarta', Journal of Industrial and Production Engineering 34(3), 199-212.

Prodhon, C. and Prins, C. (2014), 'A survey of recent research on location-routing problems', European Journal of Operational Research 238(1), 1-17.

Rescio, G., Leone, A. and Siciliano, P. (2018), 'Supervised machine learning scheme for electromyography-based pre-fall detection system', Expert Systems with Applications 100, 95-105.

Rios-Mercado, R. Z. and Escalante, H. J. (2016), 'GRASP with path relinking for commercial districting', Expert Systems with Applications 44, 102-113.

Rottkemper, B., Fischer, K. and Blecken, A. (2012), 'A transshipment model for distribution and inventory relocation under uncertainty in humanitarian operations', Socio-Economic Planning Sciences 46(1), 98 - 109.

Şahin, G. and Süral, H. (2007), 'A review of hierarchical facility location models', Computers 85 Operations Research 34(8), 2310-2331. 
Sallis, J. F., Hovell, M. F., Hofstetter, C. R., Elder, J. P., Hackley, M., Caspersen, C. J. and Powell, K. E. (1990), 'Distance between homes and exercise facilities related to frequency of exercise among san diego residents.', Public Health Reports $\mathbf{1 0 5}(2), 179$.

Schmid, V. (2012), 'Solving the dynamic ambulance relocation and dispatching problem using approximate dynamic programming', European Journal of Operational Research 219(3), 611-621.

Schmid, V. and Doerner, K. F. (2010), 'Ambulance location and relocation problems with time-dependent travel times', European Journal of Operational Research 207(3), 1293-1303.

Sha, Y. and Huang, J. (2012), 'The multi-period location-allocation problem of engineering emergency blood supply systems', Systems Engineering Procedia 5, 21-28.

Shavarani, S. M., Nejad, M. G., Rismanchian, F. and Izbirak, G. (2018), 'Application of hierarchical facility location problem for optimization of a drone delivery system: A case study of Amazon Prime air in the city of San Francisco', The International Journal of Advanced Manufacturing Technology 95(9-12), 3141-3153.

Smith, H. K., Harper, P. R., Potts, C. N. and Thyle, A. (2009), 'Planning sustainable community health schemes in rural areas of developing countries', European Journal of Operational Research 193(3), 768-777.

Snyder, L. V. (2006), 'Facility location under uncertainty: a review', IIE Transactions 38(7), 547-564.

Suurmond, J., Rosenmöller, D. L., El Mesbahi, H., Lamkaddem, M. and Essink-Bot, M.-L. (2016), 'Barriers in access to home care services among ethnic minority and Dutch elderly-a qualitative study', International Journal of Nursing Studies 54, 23-35.

Tao, Z., Cheng, Y., Dai, T. and Rosenberg, M. W. (2014), 'Spatial optimization of residential care facility locations in Beijing, China: maximum equity in accessibility', International Journal of Health Geographics 13(1), 33.

Varkevisser, M., van der Geest, S. A. and Schut, F. T. (2012), 'Do patients choose hospitals with high quality ratings? empirical evidence from the market for angioplasty in the Netherlands', Journal of Health Economics 31(2), 371-378.

Vidal, T., Crainic, T. G., Gendreau, M. and Prins, C. (2013), 'A hybrid genetic algorithm with adaptive diversity management for a large class of vehicle routing problems with time-windows', Computers \& Operations Research 40(1), 475-489.

Wang, K. and Yang, Q. (2014), 'Hierarchical facility location for the reverse logistics network design under uncertainty', Journal of Uncertain Systems 8(4), 255-270.

Warszawski, A. (1973), 'Multi-dimensional location problems', Journal of the Operational Research Society 24(2), 165-179.

WHO (2011), 'Global health and aging', https://tinyurl.com/jc92puk. Online; accessed 30-August-2018.

Widener, M. J. and Horner, M. W. (2011), 'A hierarchical approach to modeling hurricane disaster relief goods distribution', Journal of Transport Geography 19(4), 821-828.

Wilson, R. S., Krueger, K. R., Arnold, S. E., Schneider, J. A., Kelly, J. F., Barnes, L. L., Tang, Y. and Bennett, D. A. (2007), 'Loneliness and risk of Alzheimer disease', Archives of General Psychiatry 64(2), 234-240.

Wu, C., Chu, H.-H. and Chen, Y.-S. (2017), 'Identifying hierarchical multiple distinct facility location problems to enhance internet service efficiency', Journal of Internet Technology 18(2), 295-312.

Zhang, A., Kang, J. and Kwon, C. (2017), 'Incorporating demand dynamics in multi-period capacitated fast-charging location planning for electric vehicles', Transportation Research Part B: Methodological 103, 5-29.

Zhen, L., Wang, K., Hu, H. and Chang, D. (2014), 'A simulation optimization framework for ambulance deployment and relocation problems', Computers and Industrial Engineering 72(0), 12 - 23.

Zimmerman, S. M. (1997), Factors influencing Hispanic participation in prostate cancer screening., in 'Oncology Nursing Forum', Vol. 24, pp. 499-504. 